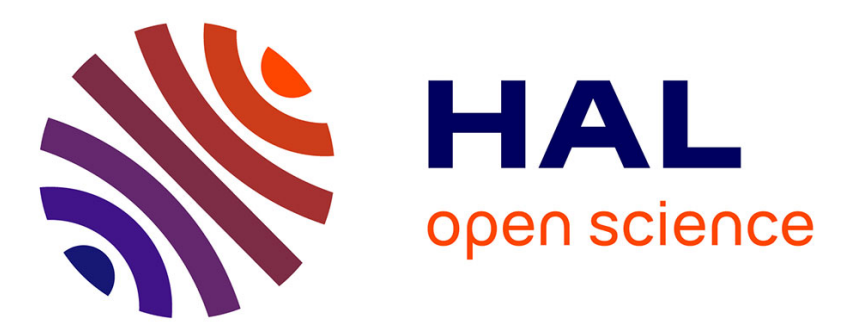

\title{
Cellules-filtres pour pompage hyperfin du 133Cs. Application à la détection optique de la transition d'horloge dans une cellule à vapeur de césium
}

\author{
M. Arditi, P. Tougne
}

\section{- To cite this version:}

M. Arditi, P. Tougne. Cellules-filtres pour pompage hyperfin du 133Cs. Application à la détection optique de la transition d'horloge dans une cellule à vapeur de césium. Revue de Physique Appliquée, 1976, 11 (5), pp.665-675. 10.1051/rphysap:01976001105066500 . jpa-00244102

HAL Id: jpa-00244102

https://hal.science/jpa-00244102

Submitted on 1 Jan 1976

HAL is a multi-disciplinary open access archive for the deposit and dissemination of scientific research documents, whether they are published or not. The documents may come from teaching and research institutions in France or abroad, or from public or private research centers.
L'archive ouverte pluridisciplinaire HAL, est destinée au dépôt et à la diffusion de documents scientifiques de niveau recherche, publiés ou non, émanant des établissements d'enseignement et de recherche français ou étrangers, des laboratoires publics ou privés. 


\title{
CELLULES-FILTRES POUR POMPAGE HYPERFIN DU ${ }^{133} \mathrm{Cs}$ APPLICATION A LA DÉTECTION OPTIQUE DE LA TRANSITION D'HORLOGE DANS UNE CELLULE A VAPEUR DE CÉSIUM
}

\author{
M. ARDITI et P. TOUGNE
}

Institut d'Electronique Fondamentale (*), Université Paris XI, 91405 Orsay, France

Laboratoire de Physique Quantique, ESPCI, 10, rue Vauquelin, 75231 Paris Cedex, France

(Reçu le 15 mars 1976, accepté le 4 mai 1976)

\begin{abstract}
Résumé. - Avec des cellules-filtres de césium + gaz tampons, utilisant les raies $D_{1}$ et $D_{2}$ sans polariseurs, on peut augmenter d'une manière très appréciable la détection optique de la transition d'horloge dans une cellule-test à vapeur de césium opérant à des températures entre 20 et $40^{\circ} \mathrm{C}$. Une théorie phénoménologique de ce filtrage donne un bon accord avec la plupart des résultats expérimentaux.
\end{abstract}

Abstract. - With filter-cells using cesium vapor and buffer gases, the optical detection of the 0-0 transition in a cesium gas cell can be greatly enhanced in the $20-40{ }^{\circ} \mathrm{C}$ temperature range. A phenomenological theory of the filtering is in qualitative agreement with most experimental results.

1. Introduction. - Le pompage optique hyperfin des alcalins a donné lieu à de nombreuses études tant du point de vue théorie que des applications. En particulier, la réalisation de certaines horloges atomiques ou de masers est basée, en grande partie, sur la production par pompage optique d'une inégalité de populations importante entre les sous-niveaux hyperfins 0-0 de l'état fondamental des atomes alcalins.

Avec la mise au point de lasers accordables en longueur d'onde, il devient possible, pour produire cette inégalité de populations, de pomper optiquement d'une façon sélective un seul des niveaux hyperfins. De tels lasers, soit à semi-conducteur du type GaAs [1], soit à colorants du type coumarine [2], ont déjà été utilisés en continu, ou en impulsions, pour le pompage hyperfin du césium. Cependant, pour certaines applications, ces lasers nécessitent, en général, un dispositif assez complexe de stabilisation de fréquence et, de ce point de vue, les lampes spectrales ordinaires retiennent un certain intérêt pratique.

A cet effet, dans le cas du rubidium, on a pu utiliser avec succès la coïncidence d'une des composantes hyperfines des isotopes 85 et 87 pour pomper sélectivement, directement, un des niveaux hyperfins ou pour réaliser des dispositifs dans lesquels les atomes d'un isotope dans une cellule servent de filtre hyperfin pour la lumière de résonance de l'autre isotope [3].

(*) Laboratoire associé au C. N. R.S.
Malheureusement cette méthode n'est pas applicable au césium qui ne possède qu'un isotope stable. Dans le passé plusieurs autres méthodes ont été proposées pour le pompage hyperfin du césium. Par exemple, on a utilisé la proximité d'une des raies de l'argon à $8521,4 \AA$ en plaçant une lampe à décharge d'argon dans un champ magnétique d'environ $5000 \mathrm{G}$ [4]. Plus récemment, des filtres hyperfins ont été réalisés en plaçant des cellules à vapeur de césium dans des champs magnétiques intenses [5-6]. Nous avions aussi proposé, pour obtenir un filtrage hyperfin, de combiner les effets d'un champ magnétique et de gaz tampons sur les déplacements du centre d'absorption des raies de résonance dans une cellule à vapeur de césium [7]. Cette étude a été reprise récemment et nous donnons ici les résultats obtenus dans l'application de tels filtres à la détection optique de la transition $F=4$, $m_{\mathrm{F}}=0 \leftrightarrow F=3, m_{\mathrm{F}}=0$, dite transition d'horloge, dans une cellule à vapeur de césium.

2. Pompage optique hyperfin du césium 133. 2.1 CONSIDÉRATIONS THÉORIQUes. - Les conditions du pompage optique hyperfin des alcalins sont très différentes selon que le temps moyen libre entre deux collisions désorientantes est beaucoup plus grand, ou beaucoup plus petit que la vie moyenne de l'état excité. La première cond tion correspond, par exemple, au cas des atomes de cérium dans une cellule à vide ou dans un jet atomique dans le vide, et la deuxième condition 
à celui des atomes de césium dans une cellule en présence de gaz tampons, à une pression relativement élevée (supérieure à quelques torrs).

Dans le premier cas, un calcul théorique [8] montre que si $D_{1}$ et $D_{2}$ ont la même intensité, l'utilisation de lumière résonante, polarisée ou non, n'a que peu d'effet sur la différence des populations des états $F=4$, $m_{\mathrm{F}}=0$ et $F=3, m_{\mathrm{F}}=0$ par rapport à cette différence, d'ailleurs très faible, en l'absence de pompage optique.

Dans le deuxième cas, par contre, la radiation réémise est complètement dépolarisée et les atomes retombent avec une égale probabilité sur tous les niveaux Zeeman de l'état fondamental, l'efficacité du pompage optique ne dépendant plus alors que du taux d'absorption de la lumière d'excitation par les différents niveaux Zeeman de l'état fondamental. Nous verrons que dans ce cas, un effet d'autofiltration peut se produire déjà dans des cellules épaisses, ce qui permet avec certains gaz tampons d'obtenir de bons signaux de détection, même avec de la lumière de résonance non filtrée.

Une théorie phénoménologique montrera également la sensibilité de la détection optique de la transition 0-0 à l'intensité de la lumière excitatrice et à sa composition spectrale.

Dans cette méthode de détection, la cellule de césium est illuminée simultanément par de la lumière de résonance et par une micro-onde à $9192 \mathrm{MHz}$. La lumière d'excitation sert aussi de lumière de détection, l'intensité lumineuse transmise diminuant lorsque le signal micro-onde passe par la résonance. Dans ces conditions, en reprenant essentiellement les analyses déjà décrites pour le cas du sodium [9] ou du rubidium [10], on peut facilement établir les équations de pompage donnant les variations des intensités des composantes hyperfines de la lumière de pompage traversant la cellule de césium.

Supposons, par exemple, $I_{3}$ et $I_{4}$ deux composantes de la lumière de résonance susceptibles d'exciter les atomes de césium des deux niveaux hyperfins $F=3$ et $F=4$. Si $a_{1}$ et $a_{8}$ représentent les fractions de populations de chacun des sous-niveaux Zeeman de $F=3$ et $F=4$, on a :

$$
7 a_{1}+9 a_{8}=1
$$

$$
\begin{aligned}
& \frac{\mathrm{d} I_{3}}{\mathrm{~d} x}=-7 k a_{1} I_{3} \\
& \frac{\mathrm{d} I_{4}}{\mathrm{~d} x}=-9 k a_{8} I_{4}
\end{aligned}
$$

$k$ : coefficient d'absorption en $\mathrm{cm}^{-1}$,

$x$, représentant l'épaisseur de la cellule d'absorption.

A l'aide de ce système d'équations on peut en déduire les variations de $I_{3}$ et $I_{4}$ en fonction de $x$. Plus généralement, si on suppose que la lumière d'excitation contient toutes les composantes hyperfines des raies $D_{1}$ et $\mathrm{D}_{2}$, désignées par $I_{3}, I_{4}$ et $J_{3}, J_{4}$, on obtient aisément les équations suivantes (en tenant compte de la relaxation) :

a) En dehors de la résonance micro-onde, on a :

$$
a_{1}=a_{2} \ldots=a_{7} \quad \text { et } \quad a_{8}=a_{9} \ldots=a_{16}
$$

ce qui donne à l'équilibre :

$$
\begin{array}{r}
a_{1}\left(I_{3}+2 J_{3}+\alpha\right)=a_{8}\left(I_{4}+2 J_{4}+\alpha\right) \\
\frac{\mathrm{d} I_{4}}{\mathrm{~d} y}=\frac{-9 I_{4}\left(I_{3}+2 J_{3}+\alpha\right)}{9\left(I_{3}+2 J_{3}\right)+7\left(I_{4}+2 J_{4}\right)+16 \alpha}= \\
=\frac{-9 I_{4}\left(I_{3}+2 J_{3}+\alpha\right)}{D} \\
\frac{\mathrm{d} I_{3}}{\mathrm{~d} y}=\frac{-7 I_{3}\left(I_{4}+2 J_{4}+\alpha\right)}{D} \\
\frac{\mathrm{d} J_{4}}{\mathrm{~d} y}=\frac{-18 J_{4}\left(I_{3}+2 J_{3}+\alpha\right)}{D} \\
\frac{\mathrm{d} J_{3}}{\mathrm{~d} y}=\frac{-14 J_{3}\left(I_{4}+2 J_{4}+\alpha\right)}{D}
\end{array}
$$

b) A la résonance micro-onde, on sature la transition $0-0$; on a :

$$
\begin{aligned}
& a_{4}=a_{12} \\
& a_{1}=a_{2}=a_{3}=a_{5}=a_{6}=a_{7}
\end{aligned}
$$

et

$$
a_{8}=a_{9}=a_{10}=a_{11}=a_{13}=a_{14}=a_{15}=a_{16}
$$

ce qui donne à l'équilibre :

$$
\begin{gathered}
6 a_{1}+2 a_{4}+8 a_{8}=1 \\
a_{1}\left(I_{3}^{\prime}+2 J_{3}^{\prime}+\alpha\right)=a_{8}\left(I_{4}^{\prime}+2 J_{4}^{\prime}+\alpha\right) \\
2 a_{1}\left(I_{3}^{\prime}+2 J_{3}^{\prime}+\alpha\right)=a_{4}\left(I_{3}^{\prime}+2 J_{3}^{\prime}+I_{4}^{\prime}+2 J_{4}^{\prime}+2 \alpha\right) \\
\frac{\mathrm{d} I_{4}^{\prime}}{\mathrm{d} y}=\frac{-I_{4}^{\prime}\left[4\left(I_{3}^{\prime}+2 J_{3}^{\prime}+\alpha\right)+5\left(I_{4}^{\prime}+2 J_{4}^{\prime}+\alpha\right)\left(I_{3}^{\prime}+2 J_{3}^{\prime}+\alpha\right)\right]}{4\left(I_{3}^{\prime}+2 J_{3}^{\prime}+\alpha\right)^{2}+3\left(I_{4}^{\prime}+2 J_{4}^{\prime}+\alpha\right)^{2}+9\left(I_{3}^{\prime}+2 J_{3}^{\prime}+\alpha\right)\left(I_{4}^{\prime}+2 J_{4}^{\prime}+\alpha\right)}=-I_{4}^{\prime} \times \frac{A}{\Delta} \\
\frac{\mathrm{d} I_{3}^{\prime}}{\mathrm{d} y}=\frac{-I_{3}^{\prime}\left[3\left(I_{4}^{\prime}+2 J_{4}^{\prime}+\alpha\right)+4\left(I_{3}^{\prime}+2 J_{3}^{\prime}+\alpha\right)\left(I_{4}^{\prime}+2 J_{4}^{\prime}+\alpha\right)\right]}{\Delta}=-I_{3}^{\prime} \times \frac{B}{\Delta}
\end{gathered}
$$




$$
\begin{aligned}
& \frac{\mathrm{d} J_{4}^{\prime}}{\mathrm{d} y}=-2 J_{4}^{\prime} \times \frac{A}{\Delta} \\
& \frac{\mathrm{d} J_{3}^{\prime}}{\mathrm{d} y}=-2 J_{3}^{\prime} \times \frac{B}{\Delta}
\end{aligned}
$$

avec

$$
y=\frac{\pi \Gamma_{0} c N f x}{\delta v_{\mathrm{D}}}
$$

et

$$
\alpha=\frac{1}{\pi \Gamma_{0} f c T I_{0}}
$$

$I_{0} \quad$ étant l'intensité de la composante $F=4$ de $\mathrm{D}_{1}$ et $\delta v_{\mathrm{D}}$ la largeur Doppler de cette composante,

$f$ la force d'oscillateur de la composante $6 \mathrm{P}_{1 / 2}$, $F=4 \rightarrow 6 \mathrm{~S}_{1 / 2}, F=4$, de $\mathrm{D}_{1}$. (Dans les équations on a tenu compte du fait que la force d'oscillateur pour la raie $\mathrm{D}_{2}$ est double de celle de $\mathrm{D}_{1}$,)

$x$ l'épaisseur de la cellule d'absorption et $N$ le nombre d'atomes de césium par $\mathrm{cm}^{3}$,

$\Gamma_{0}$ le rayon classique de l'électron, $c$ la vitesse de la lumière,

$T \quad$ le temps de relaxation des atomes de césium dans la cellule avec gaz tampons,

$I_{3}=I_{3} / I_{0}, I_{4}=I_{4} / I_{0}$, les intensités réduites des composantes hyperfines de $\mathrm{D}_{4}$,

$J_{3}$ et $J_{4}$ les valeurs correspondantes pour les composantes hyperfines de $\mathrm{D}_{2}$.

Le signal de détection optique $S_{\mathrm{oo}}$, provenant de la saturation des niveaux $m_{\mathrm{F}}=0$ à la résonance microonde, correspond à la différence d'absorption des composantes hyperfines dans les deux cas $a$ et $b$,

$$
S_{\mathrm{oo}}=\left(I_{3}+I_{4}+J_{3}+J_{4}\right)-\left(I_{3}^{\prime}+I_{4}^{\prime}+J_{3}^{\prime}+J_{4}^{\prime}\right) \text {. }
$$

Le paramètre $y$ dépend en particulier de $N$ et de $x$. Pour une cellule de longueur donnée, $N$ sera fonction de la température $\theta$ de la cellule. On peut donc considérer $y$ comme lié à la température de la cellule (on peut, par exemple, relier $N$ et la température $\theta$ de la cellule par la formule de Langmuir-Taylor [11]) :

$$
\log _{10} N=29,531-2 \log _{10} \theta-(4150 / \theta)
$$

pour

$$
\theta<302 \mathrm{~K}
$$

et

$$
\log _{10} N=30,0379-2,35 \log _{10} \theta-(4041 / \theta)
$$

pour

$$
\theta>302 \mathrm{~K}
$$

en prenant $f=0,179$ pour la raie $\mathrm{D}_{1}$ [12], $x=3,5 \mathrm{~cm}$ et $\delta v_{\mathrm{D}}=1800 \mathrm{MHz}$ environ (pour tenir compte de la structure hyperfine de l'état $\left.6 \mathrm{P}_{1 / 2}\right)$, on obtient :

$$
y=0,925 \times 10^{-11} \mathrm{~N} .
$$

Les paramètres $T$ et $I_{0}$ n'étant connus que par leur ordre de grandeur nous les avons ajustés une fois pour toute de façon que le maximum du signal $S_{\text {oo }}$ corresponde à la température de $50^{\circ} \mathrm{C}$ mesurée expérimentalement dans le cas de la lumière non filtrée.

Par ailleurs, le temps de relaxation $T$ variant avec la température, et par suite avec $N$, nous avons adopté la loi de variation suivante, en accord avec la référence [13]

$$
\frac{1}{T}=153+8 N \times 10^{-10} \mathrm{~s}^{-1}
$$

soit pour $I_{0}=2 \times 10^{4}$ photons $/ \mathrm{cm}^{2}$.s.s ${ }^{-1}$ et $N=4,25 \times 10^{11}\left(\right.$ à $\left.50^{\circ} \mathrm{C}\right) \mathrm{cm}^{-3}$

$$
\alpha \approx 5 \quad \text { et } \quad y \approx 4 \text {. }
$$

Le système des 8 équations différentielles (5) à (12) a été programmé sur ordinateur IBM 1130 afin de calculer les variations du signal $S_{\mathrm{oo}}$ en fonction de $y$, c'està-dire de la température $\theta$, pour différentes compositions spectrales $I_{3}, I_{4}, J_{3}$ et $J_{4}$ de la lumière de pompage. La température étant le facteur expérimental le plus facile à faire varier on a surtout étudié ici les variations de $S_{\mathrm{oo}}$ en fonction de la température de la cellule-filtre ou de la cellule-test. Par exemple, sur la figure $1 A$, on a représenté les valeurs calculées de $S_{\text {oo }}$ en fonction de la température de la cellule-test, pour une lumière incidente dont les composantes spectrales auraient les intensités suivantes: $I_{3}=I_{4}=1$ pour $\mathrm{D}_{1}$ et $J_{3}=J_{4}=0,5$ pour $\mathrm{D}_{2}$. Egalement sur cette figure, on a tracé les courbes de $S_{\mathrm{oo}}$ correspondant à des intensités incidentes plus faibles, mais de même composition spectrale (dans ce cas un filtre absorbant neutre est intercalé entre la lampe d'excitation et la cellule test). On voit que le maximum de $S_{\text {oo }}$ est en gros proportionnel à la puissance 2 de l'intensité de la lumière de pompage.

Le calcul permet aussi d'obtenir les signaux $S_{\text {oo }}$ en fonction de la température de la cellule-test, pour différentes conditions de pompage optique ou de détection. Par exemple, sur la figure $1 B$, outre la courbe (1) obtenue précédemment pour le pompage et la détection avec les deux composantes $D_{1}$ et $D_{2}$, on a tracé les courbes suivantes : les courbes (2) et (3) correspondent 

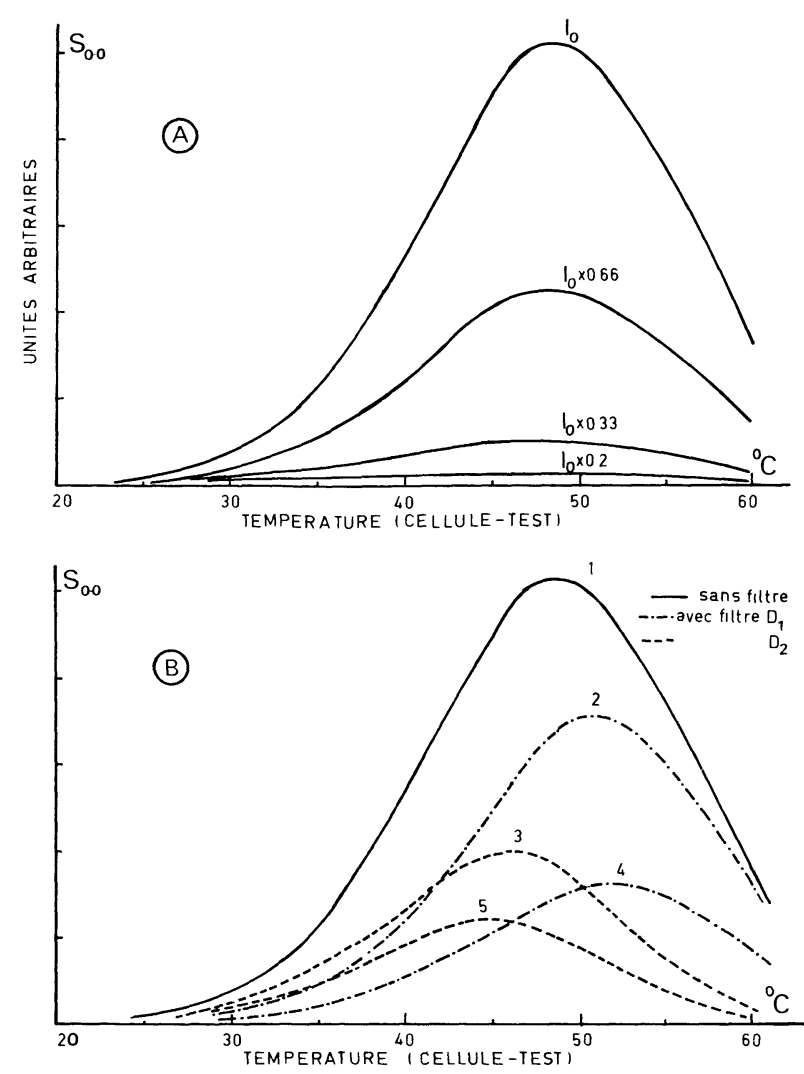

Fig. 1A. - Courbes théoriques de $S_{\text {oo }}$ en fonction de la température de la cellule-test pour différentes intensités de la lumière incidente.

FIG. 1B. - Courbes (1) sans filtre, (2) et (3) avec filtres $\mathrm{D}_{1}$ ou $\mathrm{D}_{2}$ derrière la cellule-test, (4) et $(5)$ avec filtres $D_{1}$ ou $D_{2}$ devant la cellule-test.

à un pompage optique avec les deux composantes $\mathrm{D}_{1}$ et $\mathrm{D}_{2}$ mais avec une détection du signal de la composante $D_{1}$ ou $D_{2}$ seulement (dans ce cas on place un filtre interférentiel, correspondant à $\mathrm{D}_{1}$ ou $\mathrm{D}_{2}$, entre la cellule-test et la cellule photo-électrique); les courbes (4) et (5) correspondent à un pompage optique et à une détection pour les composantes $\mathrm{D}_{1}$ ou $\mathrm{D}_{2}$ seules (en plaçant des filtres interférentiels passant $\mathrm{D}_{4}$ ou $\mathrm{D}_{2}$ devant la cellule-test).

On peut aussi calculer le signal $S_{\text {oo }}$ obtenu en envoyant sur la cellule-test de césium, une lumière de résonance préalablement filtrée par une cellule-filtre et donnant ainsi une certaine composition spectrale à la lumière d'excitation. Par exemple, sur la figure 2, outre la courbe (1) correspondant à $I_{3}=I_{4}=1$ et $J_{3}=J_{4}=0,5$ on a tracé la courbe (2) pour $I_{3}=0,6, I_{4}=0,428$ et $J_{3}=0,214, J_{4}=0,153$, et la courbe (3) pour $I_{3}=0,4, I_{4}=0,2424$ et $J_{3}=0,133$, $J_{4}=0,095$, enfin (4) pour $I_{3}=0,25, I_{4}=0,1315$ et $J_{3}=0,0781, J_{4}=0,06$. Ces courbes correspondent à la composition spectrale de la lumière à la sortie de la cellule-filtre $\left(\mathrm{Cs}+5\right.$ torrs $\left.\mathrm{N}_{2}, H_{0}=700 \mathrm{G}\right)$ pour des températures de $43,47{ }^{\circ} \mathrm{C}$ et $55^{\circ} \mathrm{C}$ et seront à comparer avec les résultats expérimentaux de la figure 9.

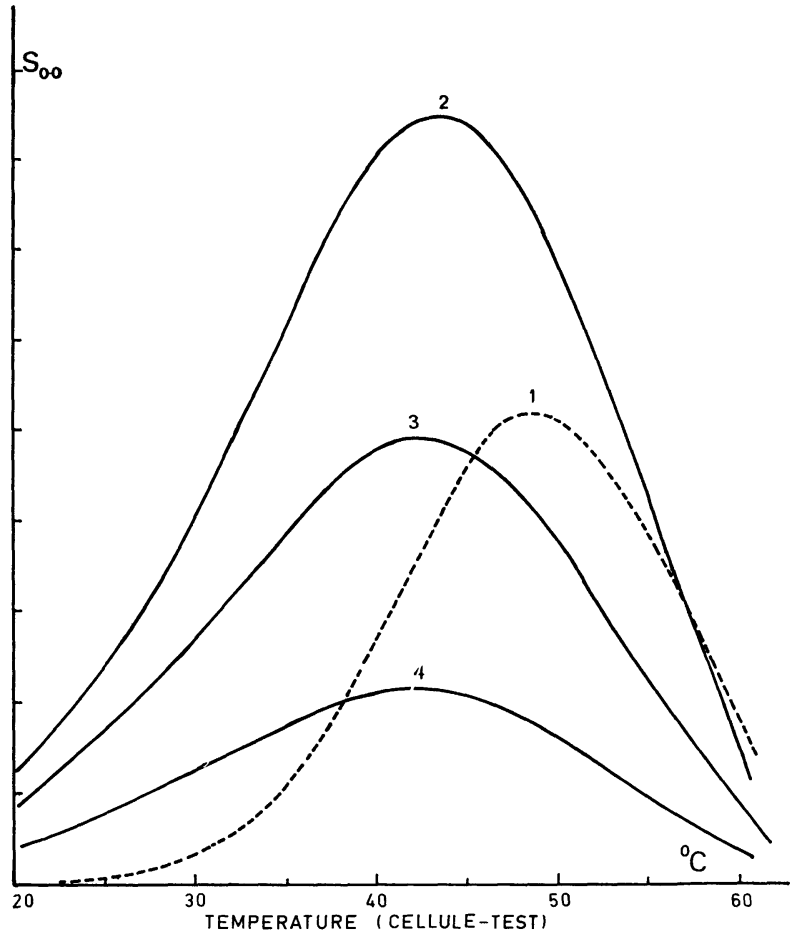

Fig. 2. - Courbes théoriques de $S_{\mathrm{oo}}$ en fonction de la température de la cellule-test : (1) sans cellule-filtre, (2), (3) et (4) avec cellule-filtre à différentes températures $\left[(2)=43^{\circ} \mathrm{C}\right.$; (3) $\left.=47^{\circ} \mathrm{C} ;(4)=55^{\circ} \mathrm{C}\right]$.

Remarquons que les valeurs de $S_{\mathrm{oo}}$ ainsi obtenues correspondent à des pourcentages de variations de la lumière incidente relativement faibles (quelques $0,1 \%$ au plus) et les calculs doivent être conduits avec suffisamment de précision pour être significatifs.

On voit que l'action de la cellule-filtre dans ce cas est de déplacer le maximum de $S_{\mathrm{oo}}$ vers les basses températures, donnant ainsi un gain très appréciable $\mathrm{du}$ signal $S_{\text {oo }}$ pour des températures de la cellule-test inférieures à $25-30{ }^{\circ} \mathrm{C}$.

Dans les calculs précédents, nous avons négligé les effets de la diffusion multiple, ce qui est justifié quand on considère le cas de cellules de césium contenant de l'azote comme gaz tampon. En effet, pour ce gaz il y a un effet de quenching très prononcé, en particulier, aux températures élevées, et il n'y a pratiquement pas de lumière de fluorescence réémise pouvant s'opposer au pompage optique.

On a d'abord mesuré $S_{\mathrm{oo}}$ pour vérifier l'effet d'autofiltration dans des cellules césium + gaz tampons, relativement épaisses, et on a ensuite évalué l'efficacité de plusieurs types de cellules-filtres en fonction de la température de la cellule-test ou de la cellule-filtre.

2.2 Montage eXPérimental. - Outre la mesure de $S_{\text {oo }}$ par détection optique de la transition 0-0 en micro-onde, on a aussi mesuré, à l'aide d'un spectromètre de Fabry-Pérot, l'intensité relative des composantes spectrales $I_{3}, I_{4}, J_{3}$ et $J_{4}$ des raies $\mathrm{D}_{1}$ et $\mathrm{D}_{2}$ dans la lumière d'excitation, avec ou sans filtrage. 
Les conditions expérimentales étaient les suivantes :

2.2.1 Lampes spectrales. — Les lampes étaient du genre bouton, en pyrex de $2 \mathrm{~cm}$ de diamètre, remplies de césium +2 torrs d'argon, et excitées en radiofréquence $(90 \mathrm{MHz})$ [14]. Avec ces lampes l'équilibre thermique s'établit assez rapidement et elles ont une intensité remarquablement 'stable, permettant des mesures comparatives sur plusieurs heures. A l'allumage, lorsque les lampes sont encore relativement froides, le rapport des intensités des raies $D_{1}$ et $D_{2}$ est de l'ordre de 1/2. Mais quand l'équilibre thermique s'établit ce rapport s'inverse et devient très voisin de 2 , avec un rapport des composantes $I_{3} / I_{4}$ ou $J_{3} / J_{4}$ très proche de 1. On n'a pas mesuré l'intensité absolue de la lumière de résonance émise par ces lampes, mais elle peut être estimée de l'ordre de $10^{5}$ à $10^{4}$ photons $/ \mathrm{cm}^{2} . \mathrm{s} . \mathrm{s}^{-1}$, selon des mesures faites ailleurs sur des lampes de ce type [10].

2.2.2 Cellules-tests de césium. - Ce sont des cellules cylindriques, à faces planes-parallèles en pyrex de $5 \mathrm{~cm}$ de diamètre et de $3,5 \mathrm{~cm}$ de longueur, remplies de césium et d'un gaz tampon. On a surtout étudié ici comme gaz tampons, l'azote et le néon, à des pressions de 5 à 6 torrs. Cela nous a permis de mettre en évidence l'effet de quenching de l'azote. Cet effet, en réduisant la lumière de fluorescence diminue la dépopulation des niveaux hyperfins par cette lumière, et permet ainsi d'obtenir des signaux $S_{\text {oo }}$ plus grands qu'avec le néon, par exemple, qui ne possède pas cet effet.

La température de la cellule-test était mesurée par un thermocouple placé sur le point apparemment le plus froid de la cellule. Cependant le chauffage de la cellule n'était pas très homogène, pour éviter un dépôt de césium sur les surfaces exposées à la lumière, et par suite les indications de température n'étaient reproductibles qu'au degré près.

2.2.3 L'interféromètre de Fabry-Perot. - L'interféromètre était du type à miroirs plans, de réflectivité $97,5 \%$ à $\lambda=9000 \AA$. La distance entre les miroirs étaient de 7,14 mm. La finesse de l'appareil était de l'ordre de 10. Le balayage en fréquence se faisait à l'aide de cales piézo-électriques commandées par une rampe de voltage. Les spectres de la lumière transmise à travers les cellules-tests ou les cellules-filtres étaient obtenus en utilisant des filtres interférentiels pour les raies $\mathrm{D}_{1}$ ou $\mathrm{D}_{2}$ de $100 \AA$ de largeur à mi-hauteur avec une transmission d'environ $85 \%$. De plus, un filtre Kodak Wratten $87 \mathrm{C}$ permettait d'éliminer la lumière visible. Quelques réserves peuvent cependant être faites sur la validité des spectres obtenus étant donné la finesse relativement faible de l'interféromètre et la largeur des raies d'absorption dans les cellules de césium, surtout aux basses températures.

2.2.4 Dispositif expérimental pour la mesure de $S_{\mathrm{oo}}$. - Le montage expérimental est représenté schématiquement sur la figure 3. L'instrumentation pour l'exci-

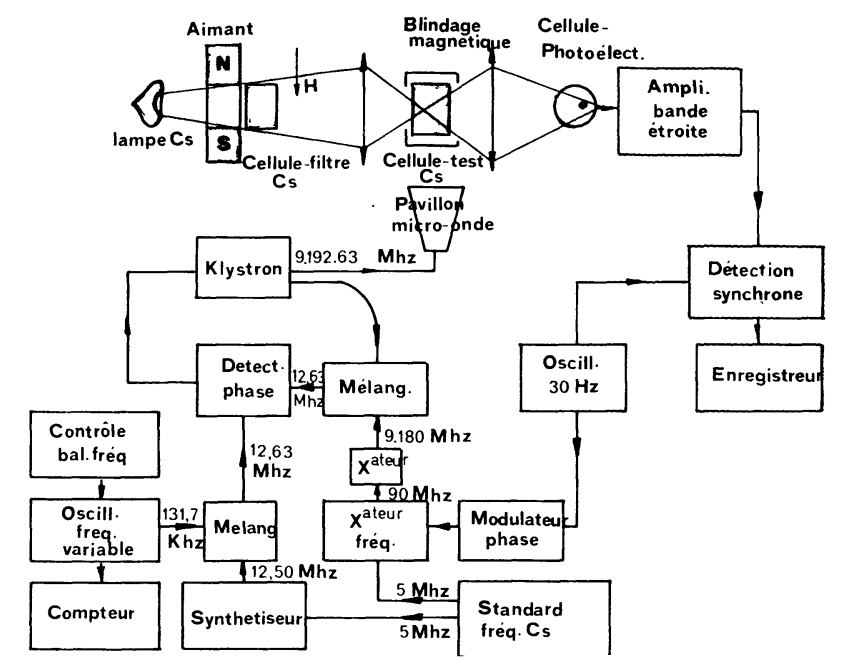

FIG. 3. - Montage expérimental pour la détection optique de la transition d'horloge dans le césium.

tation en micro-onde à $9192 \mathrm{MHz}$ et la détection optique de la transition 0-0 est assez semblable à celle déjà décrite précédemment [14]. En modulant, à basse fréquence, la fréquence de la radiation micro-onde, le courant de sortie de la cellule photo-électrique est aussi modulé et peut être détecté avec une détection synchrone. En balayant la fréquence micro-onde autour de la fréquence de résonance, on peut obtenir ainsi la dérivée de la courbe de résonance de la transition 0-0. Le signal $S_{\text {oo }}$ est alors défini, en valeur relative, par le maximum de la courbe de résonance et la largeur de la résonance 0-0 par l'intervalle de fréquence entre les maximums de la courbe dérivée. La cellule photoélectrique de détection était du type HP-5082 couplée à un amplificateur à bande étroite, l'ensemble ayant un gain dynamique très large, sans saturation.

2.2.5 Cellule-filtre de césium. - Les cellules-filtres étaient géométriquement identiques aux cellules-tests. On a utilisé surtout deux types de cellules-filtres :

- ou bien des cellules de césium remplies d'azote à la pression de 5 torrs et placées dans un champ magnétique modéré, perpendiculaire à la direction de la lumière,

- ou bien des cellules de césium remplies d'hélium à la pression de 35 torrs utilisées dans le champ magnétique terrestre.

On a également fait quelques observations à l'aide de cellules-filtres remplies des mêmes gaz à différentes pressions, ou de formes géométriques différentes, mais ces observations n'ont pas été suffisamment systématiques ou complètes pour pouvoir être reportées ici.

2.3 RÉSUltats eXPÉRIMENTAUX. - 2.3.1 Pompage hyperfin par auto-filtrage. - Rappelons que l'effet d'auto-filtrage dans des cellules à vapeur alcaline + gaz tampon, avait déjà été utilisé auparavant pour la détection optique de la transition 0-0 dans de nombreuses 
vapeurs alcalines [15] (potassium, sodium, rubidium ou césium).

Dans la présente étude on a utilisé une cellule-test contenant du césium +5 torrs d'azote, placée dans le champ magnétique terrestre. La figure $4 A$ montre la valeur du signal $S_{\text {oo }}$ en fonction de la température de la cellule, et pour différentes atténuations de la lumière incidente de composition spectrale correspondant à $I_{3}=I_{4}=1$ et $J_{3}=J_{4}=0,5$. On voit que pour une température de 45 à $50^{\circ} \mathrm{C}$ on obtient un très bon signal, avec une augmentation considérable du rapport signal/bruit, par rapport à cette valeur à $20^{\circ} \mathrm{C}$ (la grandeur du maximum du signal de modulation $S_{\text {oo }}$ correspondait approximativement, à quelques millièmes de l'intensité incidente).
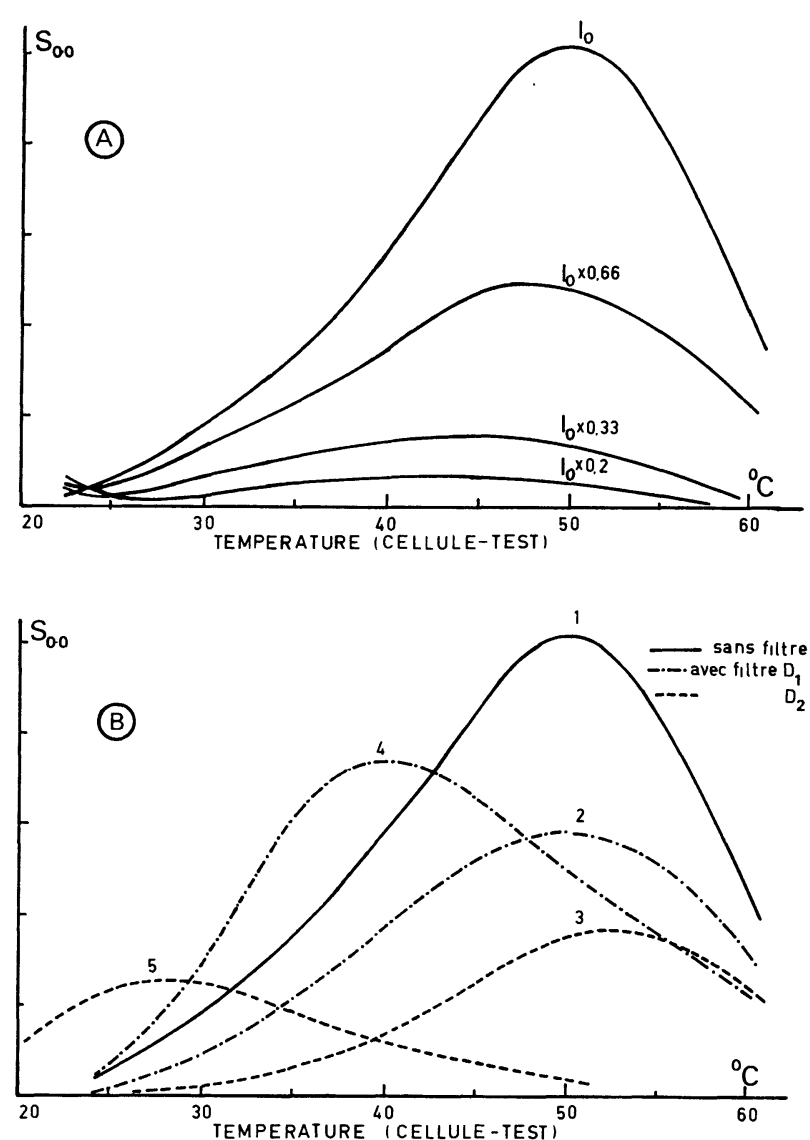

Fig. 4A. - Courbes expérimentales de $S_{\text {oo }}$ en fonction de la température de la cellule-test $\left(\mathrm{Cs}+5\right.$ torrs $\mathrm{N}_{2}$ ) pour différentes intensités de la lumière incidente.

Fig. 4B. - Courbes (1) sans filtre, (2) et (3) avec filtres $D_{1}$ ou $D_{2}$ derrière la cellule-test, (4) et (5) avec filtres $D_{1}$ ou $D_{2}$ devant la cellule-test.

Afin de pouvoir comparer les divers résultats expérimentaux, la valeur de $S_{\text {oo }}$ est donnée en unités arbitraires mais l'échelle de $S_{\mathrm{oo}}$ a été maintenue constante dans toutes les figures (sauf sur la figure 5 , cas d'une cellule césium + néon). De plus, on a normalisé les courbes des figures $1 A$ et $4 A$ correspondant à l'intensité $I_{0}$ afin de relier les calculs théoriques aux résultats expérimentaux.

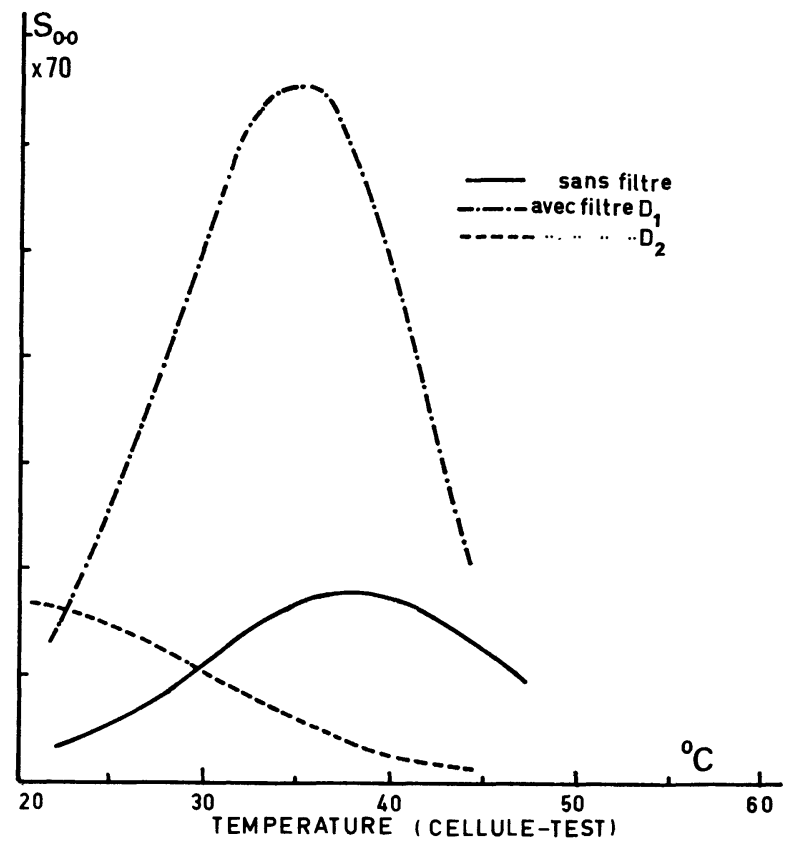

Fig. 5. - Courbe expérimentale de $S_{\text {oo }}$ en fonction de la température de la cellule-test $\left(\mathrm{Cs}+6\right.$ torrs $\mathrm{Ne}$ ) avec filtres $\mathrm{D}_{1}$ ou $\mathrm{D}_{2}$ devant la cellule-test.

Expérimentalement sur la figure $4 A$ le maximum de $S_{\mathrm{oo}}$ est proportionnel à la puissance 1,8 de l'intensité incidente, au lieu de puissance 2 trouvée théoriquement. Aux températures élevées il y a un bon accord avec les résultats théoriques; l'accord est moins bon aux basses températures mais nous donnerons une explication possible de cet effet dans la discussion. On a remarqué également que, en général, la polarisation de la lumière incidente, linéaire ou circulaire, était pratiquement sans effet sur la grandeur de $S_{\text {oo }}$.

On a étudié aussi l'effet comparatif des filtres $\mathrm{D}_{1}$ ou $\mathrm{D}_{2}$ placés soit devant la cellule-test (pompage et détection par $D_{1}$ ou $D_{2}$ seules) soit derrière la celluletest (pompage par $D_{1}+D_{2}$ et détection de $D_{1}$ ou $D_{2}$ seules). Les résultats expérimentaux de la figure $4 B$ sont à comparer avec les résultats théoriques de la figure $1 B$. En particulier on notera sur la figure $4 B$ un déplacement plus marqué du maximum de $S_{\mathrm{oo}}$ vers les basses températures surtout pour le cas du pompage avec $\mathrm{D}_{2}$ seule. Cela est peut-être dû au fait que le quenching n'est pas égal pour les raies $D_{1}$ et $D_{2}[16]$, que ce quenching est moins efficace aux basses températures, et que le phénomène de diffusion multiple pour $\mathrm{D}_{2}$ intervient à de plus basses températures que pour $\mathrm{D}_{1}$, particularités qui n'ont pas été introduites dans la théorie précédente dans un but de simplification. L'effet prépondérant du quenching est encore plus apparent quand on compare les résultats de la figure $4 B$ à ceux de la figure 5 , correspondant à une cellule de césium +6 torrs de néon, gaz qui ne produit pas de quenching appréciable, et pour laquelle le signal $S_{\mathrm{oo}}$ est plus faible dans un rapport 200.

Bien que l'auto-filtration dans une cellule césium + azote soit efficace aux températures élevées 
pour la détection de la transition $0-0$, on ne peut utiliser une telle cellule directement comme cellule-filtre devant une autre cellule-test identique. L'expérience montre en effet que ce dispositif a pour effet de réduire considérablement le signal $S_{\mathrm{oo}}$. Cela peut se comprendre si on remarque que les spectres des raies $D_{1}$ et $D_{2}$ à travers la cellule césium +5 torrs d'azote n'indiquent qu'une très faible variation, en fonction de la température de la cellule, du rapport des composantes $I_{3} / I_{4}, J_{3} / J_{4}$ ou $I_{3} / J_{3}$ (Fig. 6 et 7). Cela est également vrai dans le cas d'une cellule de césium sans gaz tampon). Etant donnée l'absorption relativement forte de la lumière à la sortie de la cellule césium + azote à $50^{\circ} \mathrm{C}$ par exemple, on conçoit alors que cette cellule ne soit pas efficace directement comme cellule-filtre.
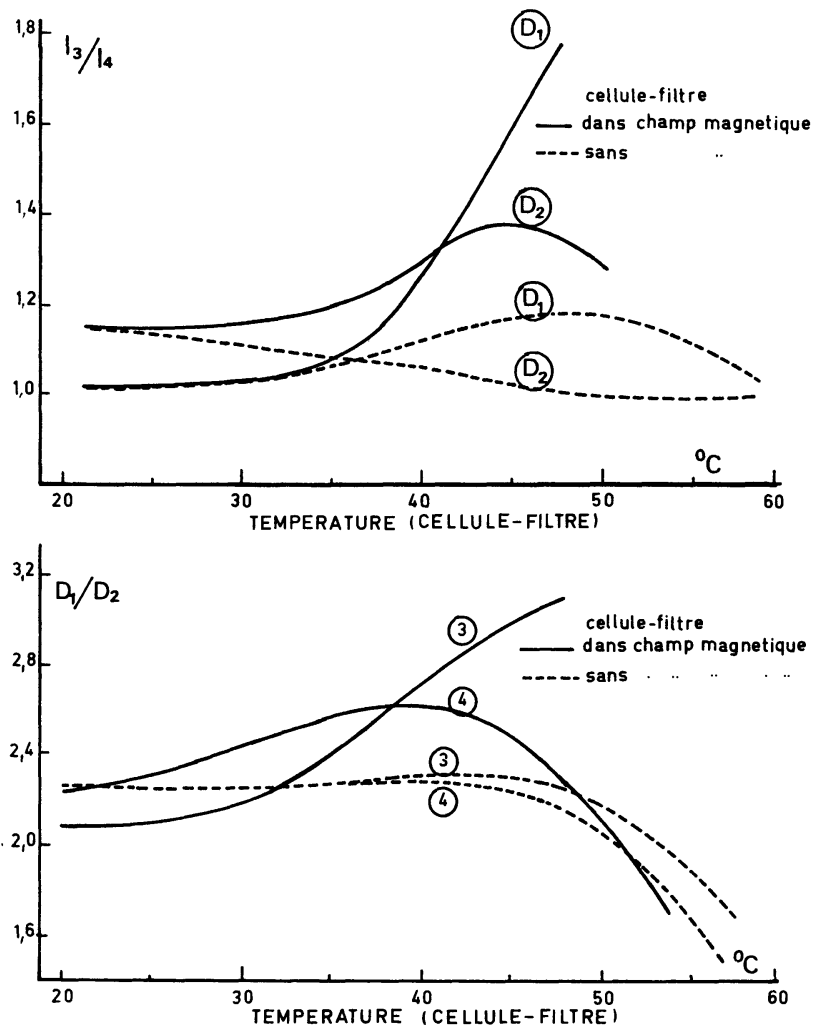

Fig. 6. $-I_{3} / I_{4}$ pour les raies $\mathrm{D}_{1}$ ou $\mathrm{D}_{2}$ en fonction de la température de la cellule-filtre $\left(\mathrm{Cs}+5\right.$ torrs $\mathrm{N}_{2}$ ) avec ou sans champ magnétique.

On peut obtenir de meilleurs résultats en combinant les effets de glissements de fréquence des composantes hyperfines du spectre d'absorption produits par les gaz tampons avec les glissements de fréquence des composantes Zeeman produits par un champ magnétique [7].

2.3.2 Pompage hyperfin avec des cellules-filtres césium + azote, dans un champ magnétique modéré. Dans une note précédente [7] nous avions indiqué qu'avec une cellule-filtre césium +52 torrs d'azote, à $32^{\circ} \mathrm{C}$, on ne changeait pratiquement pas le rapport des composantes $I_{3} / I_{4}$ quand cette cellule était placée dans un champ magnétique variant de 0 à $7 \mathrm{kG}$. Par contre, ces résultats peuvent être modifiés considé-
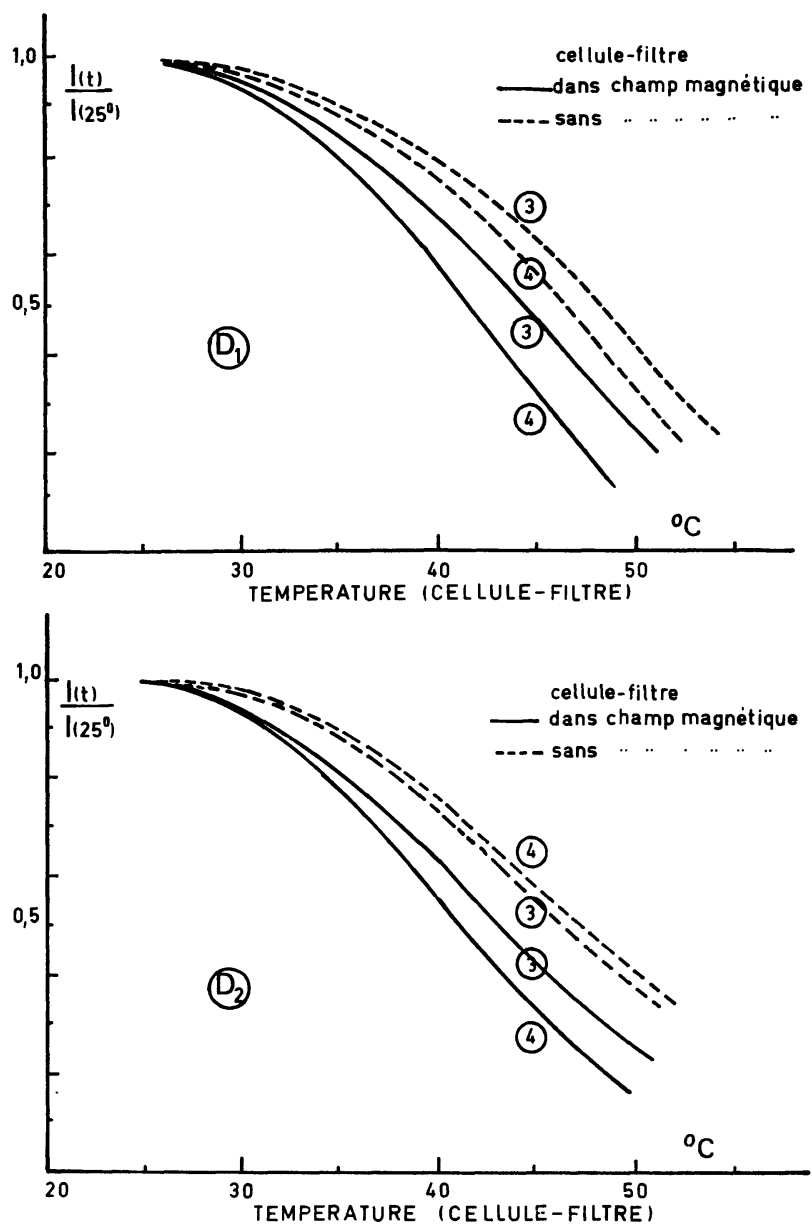

Fig. 7. $-I_{t} / I_{25}{ }^{\circ}$ pour les composantes 3 et 4 des raies $D_{1}$ ou $D_{2}$ avec ou sans champ magnétique.

rablement si la cellule-filtre, placée dans un champ magnétique modéré ( $700 \mathrm{G})$ est portée à des températures plus élevées.

Les figures 6 et 7 représentent les rapports des composantes $I_{3} / I_{4}$ ou $J_{3} / J_{4}$ de $\mathrm{D}_{1}$ et $\mathrm{D}_{2}$ déduits des spectres de Fabry-Perot de la lumière, à la sortie d'une cellule de césium +5 torrs d'azote, placée soit dans le champ terrestre, soit dans un champ magnétique de $700 \mathrm{G}$ environ. L'influence du champ magnétique est nettement marquée sur le rapport des composantes hyperfines. Les valeurs indiquées sur les courbes des figures 6 et 7 ont servi pour le calcul des courbes théoriques des figures 1 et 2 .

Les modifications de la composition spectrale de la lumière d'excitation par la cellule-filtre expliquent ainsi les résultats expérimentaux obtenus pour $S_{\text {oo }}$ avec une cellule-test contenant césium +5 torrs d'azote et placée dans le champ magnétique terrestre. Par éxemple, la cellule-test étant maintenue à une température d'environ $23^{\circ} \mathrm{C}$, le signal $S_{\text {oo }}$ passe par un maximum pour une température de la cellule-filtre de $45^{\circ} \mathrm{C}$ environ (Fig. 8). Ce signal varie aussi avec le champ magnétique, un maximum maximorum se produisant à $45-50{ }^{\circ} \mathrm{C}$ pour une valeur de $700 \mathrm{G}$ environ. Pour cette valeur du champ magnétique, la figure 9 montre le 


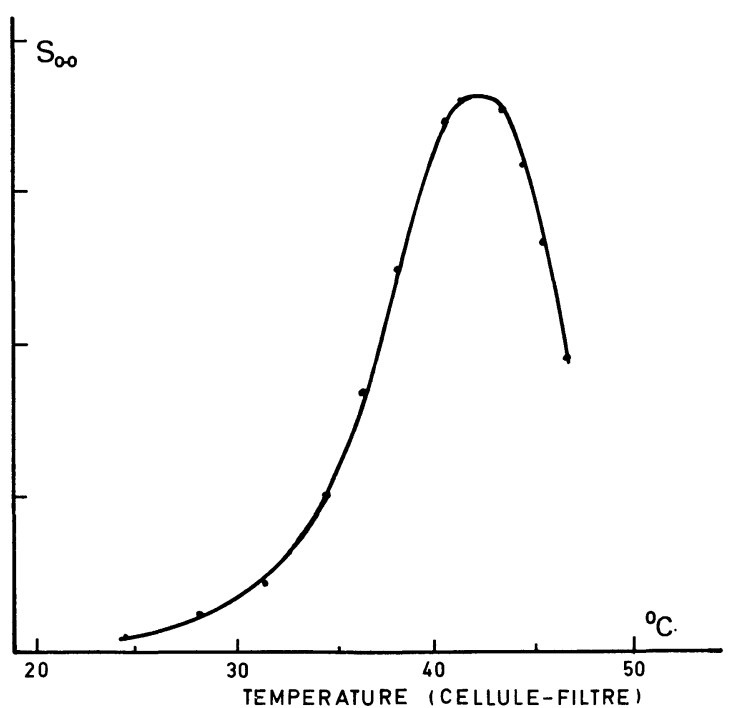

FIG. 8. $-S_{\text {oo }}$ en fonction de la température de la cellule-filtre $\left(\mathrm{Cs}+5\right.$ torrs $\left.\mathrm{N}_{2}, \mathrm{H}_{0} \approx 700 \mathrm{G}\right)$. Cellule-test : Cs +5 torrs $\mathrm{N}_{2}$ à $23{ }^{\circ} \mathrm{C}$.

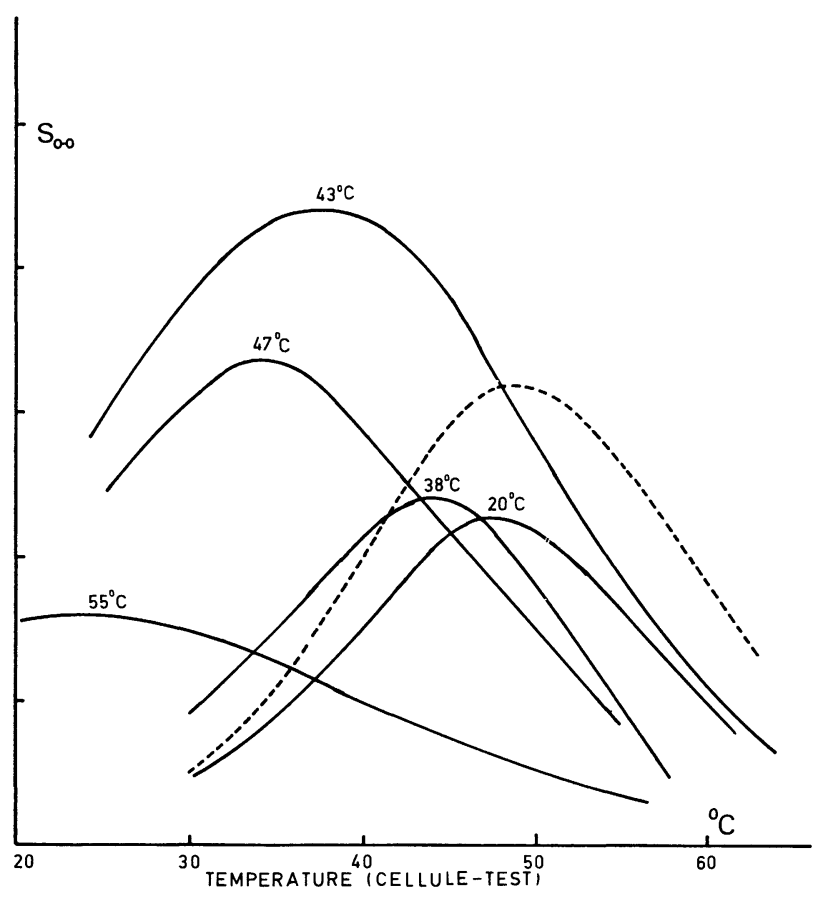

Fig. 9. $-S_{\text {oo }}$ en fonction de la température de la cellule-test (Cs +5 torrs $\mathrm{N}_{2}$ ) pour différentes températures de la cellulefiltre (Cs +5 torrs $\mathrm{N}_{2}, H_{0} \approx 700 \mathrm{G}$ ) (la courbe en tirets correspond à la cellule-test sans cellule-filtre).

signal $S_{\text {oo }}$ en fonction de la température de la celluletest et pour différentes températures fixes de la cellulefiltre.

On voit que, qualitativement, l'accord avec les courbes théoriques de la figure 2 est assez bon. En particulier, on notera que le maximum de $S_{\text {oo }}$ est nettement déplacé vers les basses températures de la celluletest.

Bien que ce genre de cellules-filtres utilise une cellule de césium + gaz tampon placée dans un champ magné- tique, son principe de fonctionnement basé sur les déplacements des raies d'absorption par les gaz tampons et un champ magnétique modéré est très différent des cellules-filtres de césium réalisées avec des champs magnétiques plus élevés utilisant l'effet Back-Goudsmit et basées sur la différence de polarisation des raies hyperfines [6]. C'est ainsi qu'un analyseur circulaire, nécessaire dans ces filtres magnétiques, placé ici entre la cellule-filtre et la cellule-test produit une baisse du signal $S_{\text {oo }}$ de $85 \%$ en l'absence de filtres $\mathrm{D}_{1}$ ou $\mathrm{D}_{2}$ et une annulation presque totale de ce signal si on ajoute un filtre $D_{1}$ ou $D_{2}$. Notons également qu'en autofiltration, la polarisation de la lumière incidente est pratiquement sans effet sur le signal $S_{\text {oo }}$ tandis qu'avec cette cellule-filtre dans un champ de $700 \mathrm{G}$, le signal $S_{\text {oo }}$ ne change pas si on place un polariseur linéaire dont la direction de polarisation est perpendiculaire à la direction du champ $H_{0}$, alors que ce signal baisse de 40 à $50 \%$ si la direction de polarisation est parallèle à $H_{0}$.

2.3.3 Pompage hyperfin avec des cellules-filtres césium + hélium. — Dans la référence [7] nous avions aussi signalé l'effet important de filtrage hyperfin produit par l'hélium dans une cellule de césium placée dans un champ faible (champ magnétique terrestre). L'hélium produit un déplacement du spectre d'absorp-

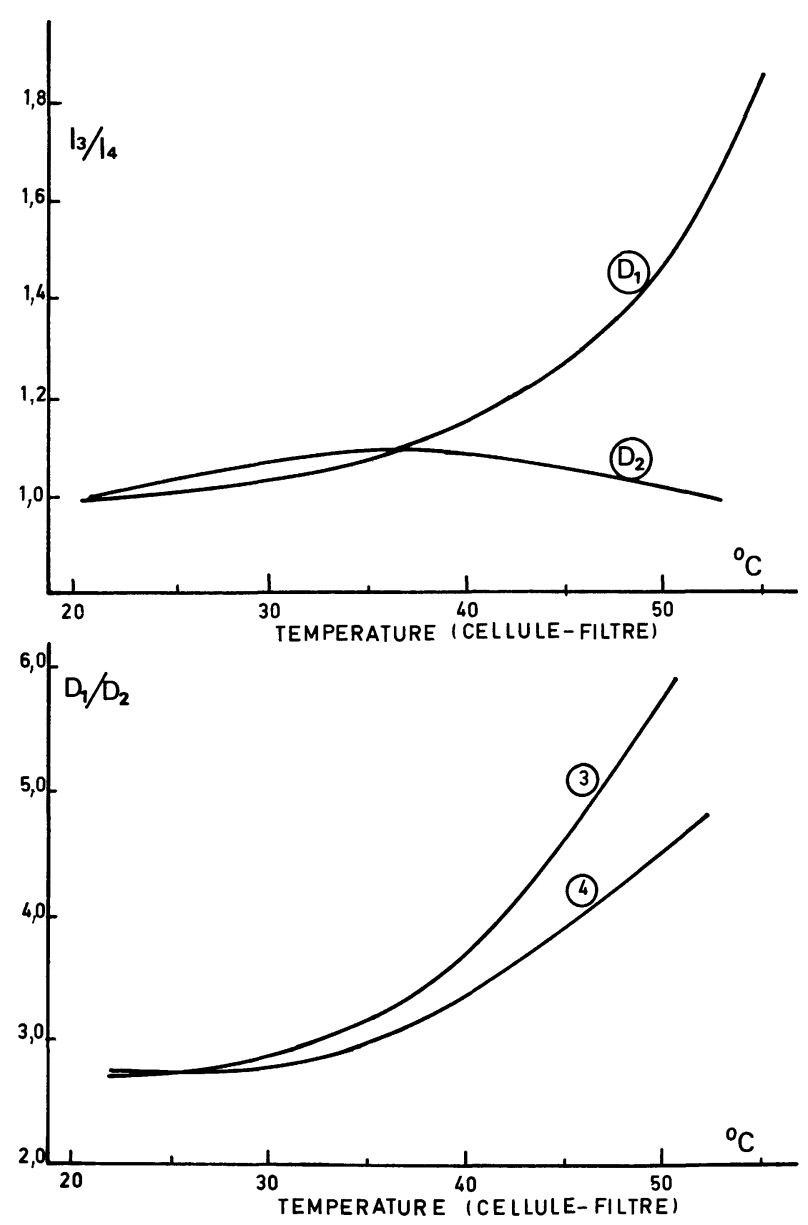

FIG. 10. - Rapport $I_{3} / I_{4}$ pour les raies $\mathrm{D}_{1}$ ou $\mathrm{D}_{2}$ en fonction de la température de la cellule-filtre ( $\mathrm{Cs}+35$ torrs $\mathrm{He}$ ). 
tion du césium vers les fréquences élevées, mais en élevant la température de la cellule-filtre il en résulte une absorption plus faible pour la composante $I_{3}$ que pour la composante $I_{4}$ de la raie $\mathrm{D}_{1}$. C'est ainsi qu'avec une cellule-filtre césium +35 torrs d'hélium, le rapport $I_{3} / I_{4}$ est égal à 1,4 à $50{ }^{\circ} \mathrm{C}$, tandis que le rapport des intensités des composantes de $D_{1}$ et $D_{2}$ peut atteindre des valeurs de 5 à 6 (Fig. 10) avec des absorptions modérées, de l'ordre de $50 \%$ à $50{ }^{\circ} \mathrm{C}$ pour la raie $\mathrm{D}_{1}$ (Fig. 11).
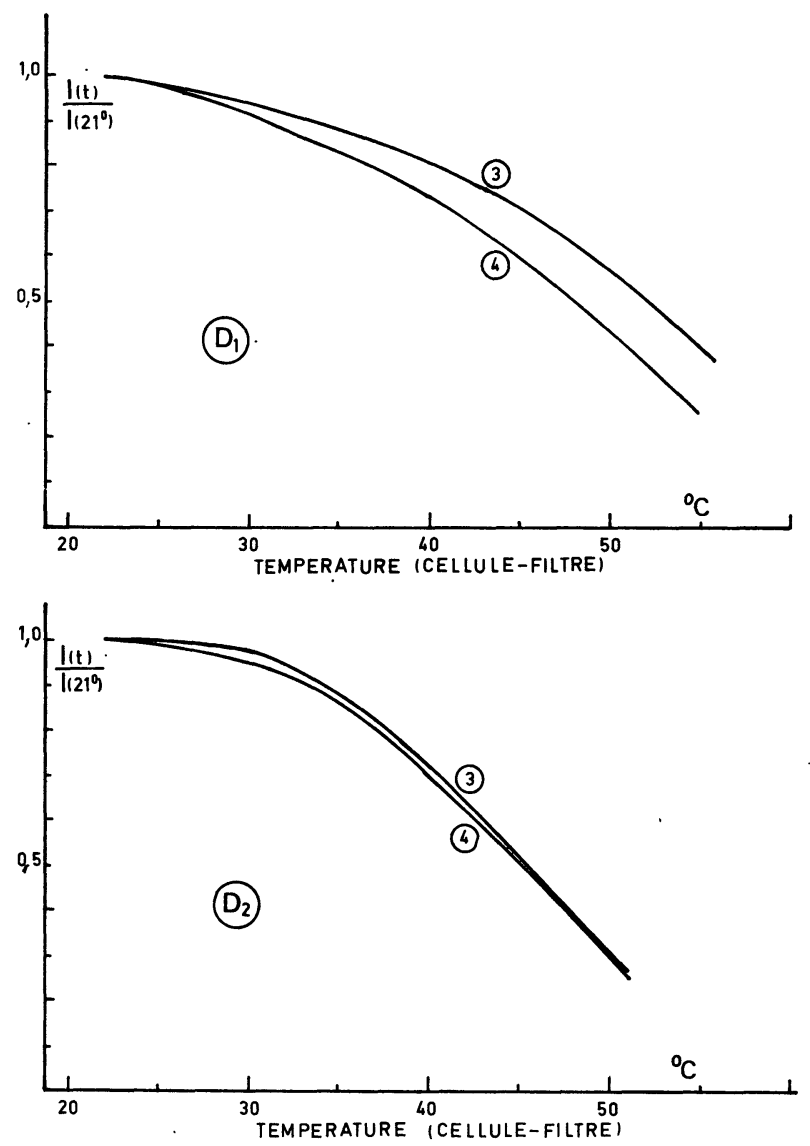

Fig. 11. - Rapport $I_{t} / I_{25^{\circ}}$ pour les composantes 3 et 4 des raies $D_{1}$ ou $D_{2}$ en fonction de la température de la cellule-filtre $(\mathrm{Cs}+35$ torrs $\mathrm{He})$.

Avec cette cellule-filtre on obtient également une augmentation considérable du signal $S_{\text {oo }}$ pour les basses températures de la cellule-test. Avec une cellule-test césium +5 torrs d'azote, la température de fonctionnement optimum de la cellule-filtre césium +35 torrs hélium se situe aux alentours de $50^{\circ} \mathrm{C}$ (Fig. 12B). Sur la figure $12 A$ on a comparé l'effet de cette celıulefiltre sur deux cellules-tests : l'une césium +5 torrs d'azote et l'autre césium +6 torrs néon, qui ont à peu près la même fréquence de résonance. Sur cette figure la courbe (1) correspond à la cellule-test césium +5 torrs azote sans cellule-filtre et la courbe (2) avec une cellule-filtre césium +35 torrs hélium dont la température a été ajustée pour obtenir le maximum de signal $S_{\mathrm{oo}}$; la courbe (3) correspond à une cellule-

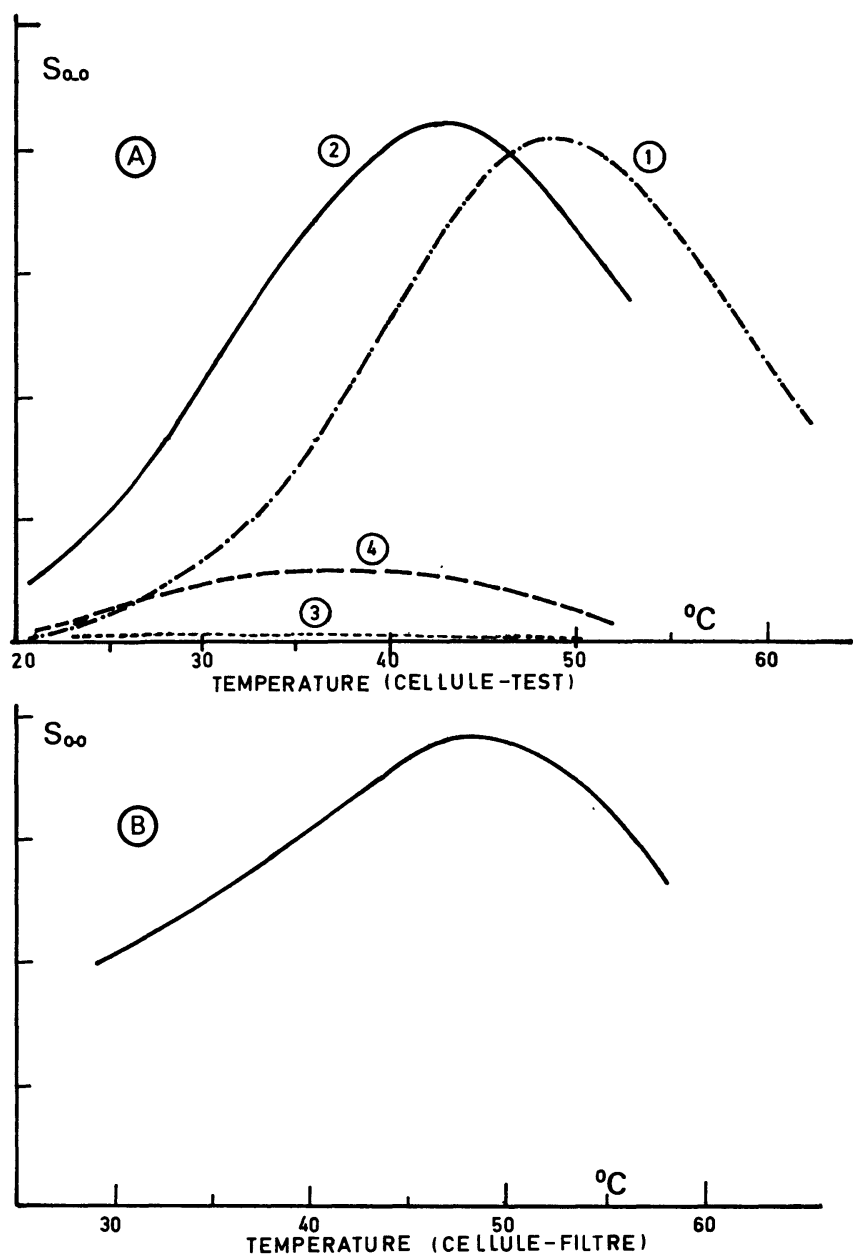

Fig. $12 A$. $-S_{\text {oo }}$ en fonction de la température de la cellule-test : a) Cellule-test : Cs +5 torrs $\mathrm{N}_{2}$ : (1) sans cellule-filtre ; (2) a vec cellule-filtre : $\mathrm{Cs}+35$ torrs $\mathrm{He}$. b) Cellule-test : $\mathrm{Cs}+6$ torrs $\mathrm{Ne}$ : (3) sans cellule-filtre ; (4) avec cellule-filtre : Cs +35 torrs He.

Fig. 12B. $-S_{\text {oo }}$ en fonction de la température de la cellule-filtre $\mathrm{Cs}+35$ torrs $\mathrm{He}$ (cellule-test à $20^{\circ} \mathrm{C}: \mathrm{Cs}+5$ torrs $\mathrm{N}_{2}$ ).

test césium +6 torrs néon sans cellule-filtre et la courbe (4) avec addition de la cellule-filtre. On peut voir sur cette figure que si l'effet de la cellule-filtre est surtout sensible aux basses températures de la celluletest césium +5 torrs azote, elle augmente considérablement le signal $S_{\mathrm{oo}}$ à toutes températures pour la cellule-test césium +6 torrs néon. On remarquera également l'effet de quenching de l'azote qui augmente beaucoup le signal $S_{\text {oo }}$ puisque, sans cellule-filtre, le rapport des maximums de $S_{\text {oo }}$ pour les deux cellulestests est de l'ordre de 200 (avec la cellule-filtre ce rapport des maximums devient égal à 7 ).

3. Discussion. - 1) Etant donné les approximations faites dans la théorie on peut considérer les résultats expérimentaux qualitativement en bon accord avec la théorie, surtout quand les effets de quenching de l'azote permettent de négliger les effets de dépolarisation par fluorescence. Ces effets sont proportionnels à l'intensité de la lumière incidente et sans quenching 
il faudrait ajouter au facteur $\alpha$ dans les équations (5) à (12), un facteur $\beta$ proportionnel à l'intensité de la lumière et dont la valeur pourrait être suffisamment élevée par rapport à $\alpha(\beta=100 \alpha$, par exemple) pour diminuer considérablement $S_{\text {oo }}$ [17].

Remarquons qu'avec la théorie phénoménologique précédente, aux basses températures, à une diminution de la lumière correspond toujours une diminution $\mathrm{du}$ signal $S_{\text {oo}}$, or, expérimentalement, nous avons observé un comportement assez différent dans un certain domaine d'intensité (Fig. 13). Ceci n'est qu'une contradiction apparente entre la théorie et l'expérience car la largeur du signal de résonance variant avec l'intensité de la lumière d'excitation (par exemple, une réduction de $70 \%$ de la lumière augmente $S_{\text {oo }}$ par un facteur 1,65 et réduit la largeur de la résonance $\delta v$ de moitié), on peut néanmoins avoir une augmentation du maximum du signal $S_{\text {oo }}$ bien que l'intégrale de celui-ci dans la largeur $\delta v$ diminue.

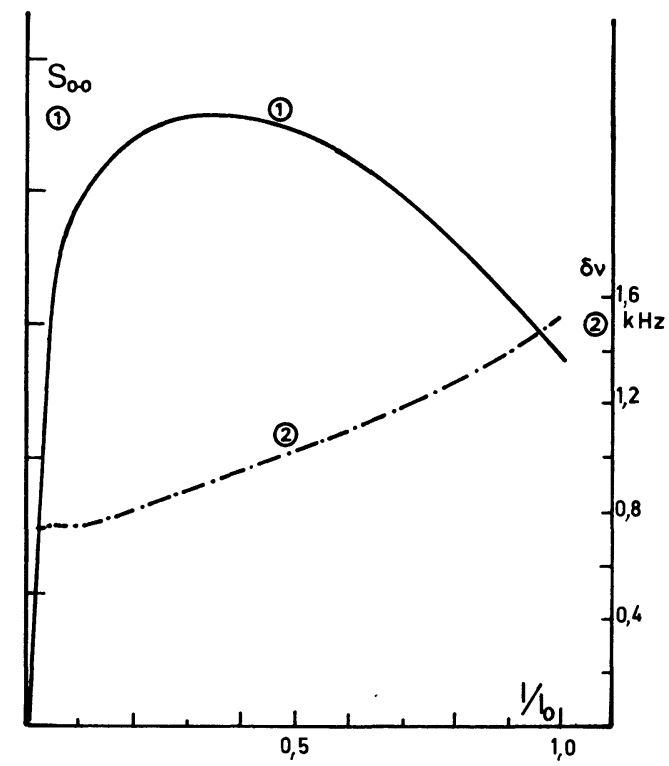

Fig. 13. - $S_{\text {oo }}$ et $\delta v$ en fonction de l'atténuation de la lumière incidente (cellule-test : $\mathrm{Cs}+5$ torrs $\mathrm{N}_{2}$ ).

L'effet de la cellule-filtre est donc favorable pour plusieurs raisons : d'une part elle produit un pompage hyperfin efficace qui augmente $S_{\text {oo }}$, et d'autre part l'absorption de la lumière par la cellule-filtre diminue $\delta v$. De plus, cette absorption de lumière réduit aussi le bruit de la détection. Il en résulte une très grande amélioration du rapport signal/bruit de la détection. Afin de rendre compte de cet effet on a représenté sur la figure 14 des enregistrements des courbes dérivées de la résonance 0-0 dans les cellules-tests avec ou sans cellules-filtres. On a également mesuré une amélioration considérable de la stabilité à court terme d'horloges atomiques à cellules de césium due à l'utilisation de ces cellules-filtres.

2) A la sortie des cellules-filtres la composante $I_{3}$ de la lumière est plus intense que la composante $I_{4}$.
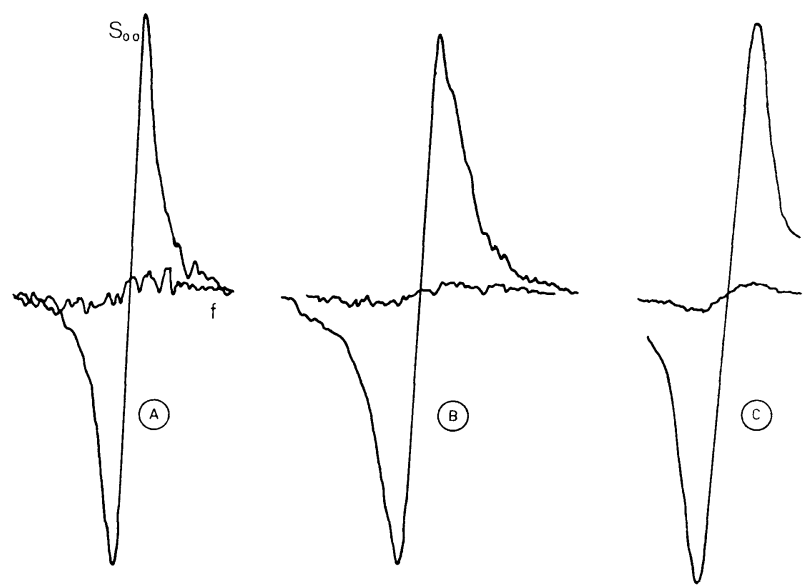

FIG. 14. - Dérivées des courbes de résonance avec ou sans cellule-filtre : A : Cellule-test : $\mathrm{Cs}+5$ torrs $\mathrm{N}_{2}\left(22^{\circ} \mathrm{C}\right)$, cellulefiltre : Cs +35 torrs $\mathrm{He}\left(45^{\circ} \mathrm{C}\right)$. B : Cellule-test : $\mathrm{Cs}+6$ torrs $\mathrm{Ne}$ $\left(45^{\circ} \mathrm{C}\right)$, cellule-filtre : $\mathrm{Cs}+35$ torrs $\mathrm{He}\left(45^{\circ} \mathrm{C}\right)$. C : Celluletest : Cs +5 torrs $\mathrm{N}_{2}\left(20^{\circ} \mathrm{C}\right)$, cellule-filtre : Cs +5 torrs $\mathrm{N}_{2}$, $H_{0} \approx 700 \mathrm{G}\left(45^{\circ} \mathrm{C}\right)$. (Dans cette figure les échelles de $S_{\mathrm{oo}}$ n'ont pas été normalisées.)

Le pompage optique hyperfin dans ces conditions a pour effet d'augmenter la population du niveau $F=4$ dans la cellule-test, produisant ainsi une inversion de populations favorable à un effet maser, dans des circonstances favorables.

3) Signalons aussi que dans le cas du pompage hyperfin du césium avec des lasers accordables, on obtient en général un meilleur rapport signal/bruit en détectant la transition 0-0 par les variations de la lumière de fluorescence plutôt que par les variations d'absorption de la lumière de pompage. Dans ces conditions, et pour les mêmes raisons de quenching, les signaux obtenus avec des cellules-tests césium +5 torrs azote sont plus faibles que ceux obtenus avec césium +6 torrs néon [17].

4) Dans les limitations de cette étude, il n'a pas été possible de déterminer les pressions optima pour l'effet des gaz dans des cellules-filtres de césium, et il n'est donc pas sûr que les résultats reportés ici représentent l'optimum de ce qui peut être obtenu avec des cellulesfiltres pour la détection optique de la transition 0-0 dans le césium. En particulier, la cellule-filtre césium + hélium qui permet de travailler sans champ magnétique, sans polariseur ni filtres interférentiels simplifie beaucoup l'usage de ces cellules-filtres, ce qui peut avoir des avantages dans certaines applications d'horloges atomiques.

4. Conclusions. - L'emploi de cellules-filtres césium + gaz tampons procure un accroissement très appréciable du rapport signal/bruit de la détection optique de la transition 0-0 dans des cellules à vapeur de césium, pour des températures de fonctionnement entre 20 et $40^{\circ} \mathrm{C}$. La simplicité de ces cellules-filtres permet d'envisager leur utilisation éventuelle dans des horloges atomiques à césium. 


\section{Bibliographie}

[1] PicQue, J.-L., IEEE Quantum Electron. QE-10 (1974) 892.

[2] Tuccio, S. A., Drexhage, K. H. et Reynolds, G. A., Opt. Commun. 7 (1973) 248.

[3] Cohen-Tannoudi, C. et Kastler, A., Progress in Optics V (1966) 10.

HAPPER, W., Rev. Mod. Physics 44 (1972) 169.

[4] Beaty, E. C., Bender, P. L. et ChI, A. R., Phys. Rev. 112 (1958) 450.

[5] Ernst, K., Minguzzi, P. et Strumia, F., Nuovo Cim. LIB (1967) 202.

[6] Beverini, N. et Strumia, F., Opt. Commun. 2 (1970) 189.

[7] Bernabeu, E., Tougne, P. et Arditi, M., C. R. Hebd. Sean. Acad. Sci. 268 (1969) 321.

[8] Hawkins, W. B., Phys. Rev. 123 (1961) 544.

[9] ВLоом, A., J. Physique Radium 19 (1958) 881.
[10] ANDres, J. M., FARmer, D. J. et INouye, G. T., IRE Trans. MIL-3 (1959) 178.

[11] TAylor, J. B. et Langmuir, I., Phys. Rev. 51 (1937) 753.

[12] Rozwadowski et Lipworth, E., J. Chem. Phys. 43 (1965) 2347.

[13] Beverini, N., Minguizzi, P. et Strumia, F., Phys. Rev. A-4 (1971) 550.

[14] Arditi, M. et Carver, T. R., Phys. Rev. 124 (1961) 800.

[15] Bell, W. E. et Bloom, E. L., Phys. Rev. 109 (1958) 219.

Arditi, M. et Carver, T. R., Phys. Rev. 109 (1958) 1012. Bloom, A. L. et CARr, J. B., Phys. Rev. 119 (1960) 1946.

[16] McGillis, D. A. et Krause, L., Phys. Rev. 153 (1967) 44.

[17] Arditi, M. et Picque, J.-L., J. Phys. B. Atom. Mol. Phys. 8 (1975) L 331. 